\title{
Incorporación de las tecnologías en la enseñanza de las matemáticas. Actitudes del estudiantado de noveno grado y educación Media
}

\author{
Belinda Fidelina Hernández Milla ${ }^{1}$ \\ Kenia Gisselle Díaz Rosales ${ }^{2}$ \\ Rebeca Yosseli Amaya Gómez ${ }^{3}$ \\ Walter Alexis Reyes Andrade ${ }^{4}$
}

\section{Información de artículo:}

Recibido: 30/03/2021

Aprobado: 30/05/2021

Palabras claves:

Actitudes, Aprendizaje, Enseñanza, Matemáticas, Tecnología.

\section{Key Words:}

Attitudes, Learning, Mathematics, Teaching, Technology

\section{Resumen}

Esta investigación ha analizado las actitudes ante la incorporación de las tecnologías en la enseñanza de las matemáticas de los estudiantes de noveno, décimo y undécimo grado. La investigación realizada es de índole cuantitativa mediante un diseño "Ex Post Facto" donde se aplicó una escala de actitudes a una muestra de 158 estudiantes de algunos centros educativos de cuatro departamentos de Honduras con edades de entre 13 a 20 años. Los resultados obtenidos indican que, en una gran mayoría, los estudiantes tienen actitudes positivas hacia el uso de la tecnología en el aprendizaje de las matemáticas independientemente de la edad y el tipo de dispositivo tecnológico a través del cual accedan a ellas. Se concluye que la incorporación de la tecnología en la enseñanza de las matemáticas resulta positiva para los estudiantes, porque una gran parte de ellos muestra satisfacción al resolver exámenes por computadora con puntuación inmediata y hacer uso de la tecnología como material de apoyo.

\footnotetext{
${ }^{1}$ Profesor de matemáticas en el grado de licenciatura. Profesor de matemáticas, Instituto Oficial "Pedro P. Amaya”. email belindamilla7@gmail.com

2 Profesor de matemáticas en el grado de licenciatura. Profesor de matemáticas, Centro de Educación Básica "Juan lindo". email keniadiazrosales94@gmail.com

3 Profesor de matemáticas en el grado de licenciatura. Profesor de matemáticas, Instituto Reyes Irene Valenzuela. email gomezrebeca144@gmail.com

4 Profesor de matemáticas en el grado de licenciatura. Profesor de matemáticas, Centro de Educación Básica Profa. Rosario Carias. email walteralexisreyesandrade@gmail.com. (D): https://orcid.org/o000-0002-2629-4663
} 


\section{Incorporating technologies in math teaching. Attitudes of the ninth-grade student and middle education.}

\section{Abstract:}

This research has analyzed the attitudes towards the incorporation of technologies in the teaching of mathematics of students in ninth, tenth and eleventh grade. The research carried out is of a quantitative nature through an "Ex Post Facto" design where an attitude scale was applied to a sample of 158 students from some educational centers in four departments of Honduras with ages between 13 and 20 years. The results obtained indicate that, in a large majority, students have positive attitudes towards the use of technology in learning mathematics regardless of age and the type of technological device through which they access them. It is concluded that the incorporation of technology in the teaching of mathematics is positive for students, because a large part of them show satisfaction in solving computerized exams with immediate scoring and making use of technology as support material.

\section{Introducción}

La incorporación de las tecnologías en la sociedad cada vez toma más relevancia, ya que hoy día en la mayor parte de las actividades de la vida cotidiana se hace uso de estas. Del mismo modo conviene mencionar la importancia de la aplicación de matemáticas en el entorno del ser humano, pues su utilidad es primordial para su desenvolvimiento e interacción. En efecto, una vida sin matemáticas seria caótica, uno de los muchos ejemplos que se puede mencionar es, que no se podría medir el tiempo y la humanidad viviría desorientada, por tal razón dicha asignatura debe ser enseñada y aprendida de forma eficiente para garantizar que los futuros profesionales tengan éxito al momento de la aplicación de esta. Esto conlleva a percibir la estrecha relación que tienen la tecnología con las matemáticas en cuanto a importancia y utilidad en la sociedad. Particularmente en la educación se ha visto la necesidad de incorporar las tecnologías, sobre todo en la enseñanza de la matemática y esto se debe a la aparición de nuevos recursos que facilitan el proceso de enseñanza-aprendizaje de la matemática, tales como la computadora el smartphone, la tableta entre otros.

Así lo afirman Salat-Figols (2013) la tecnología ha influido en la enseñanza de las matemáticas de dos maneras diferentes. Una de ellas, debido a los cambios que el quehacer matemático ha tenido con la aparición de las computadoras, que pueden procesar rápidamente grandes cantidades de datos, lo cual ha influido en la definición de los programas de las asignaturas de matemáticas. Otra, debido a que las computadoras se han convertido en un recurso para potenciar el aprendizaje. En ambos aspectos, el efecto ha ido creciendo debido a los avances en la propia tecnología computacional y a un paulatino efecto de penetración de estos recursos en la sociedad en general. 
De lo anterior resulta necesario hacer un estudio sobre las actitudes de los estudiantes ante la incorporación de la tecnología en la enseñanza de las matemáticas. Dicho estudio tiene como finalidad identificar el grado de satisfacción por parte de los estudiantes ante tal incorporación y como esta contribuye en la mejora de la enseñanza y comprensión de los temas.

\section{Literatura}

La educación no es la excepción cuando de avances tecnológicos se trata, hemos sido testigos de la incorporación de diversas técnicas de enseñanza y aprendizaje a lo largo de los años, pero a raíz de situaciones adversas en algunos casos nos hemos visto obligados a cambiar nuestra forma de enseñar o los métodos utilizados para adquirir nuevos conocimientos, tal es el caso del inicio de esta nueva década que ha traído los cambios que antes no hubiésemos imaginado por lo que ahora como nunca nos hemos visto orillados a hacer uso diversas herramientas tecnológicas para hacer llegar los conocimientos y contenidos a los alumnos en los distintos niveles académicos.

Debido al creciente desarrollo tecnológico y a la necesidad de implementar nuevas estrategias para facilitar la comprensión de los estudiantes y al aprovechamiento de los recursos a los que estos tienen acceso la realización de estudios acerca del uso de las tecnologías en la enseñanza de las matemáticas se ha vuelto una gran necesidad en la actualidad. Por otro lado, Villamizar (2018) asegura que el uso de la tecnología digital puede ser un factor motivante en la enseñanza de las matemáticas, permitiendo al estudiante la visualización de representaciones de los objetos matemáticos de manera dinámica. Sin embargo, la tecnología por sí misma no genera conocimiento, ni sustituye al profesor, por lo tanto, es necesario enmarcar las aplicaciones tecnológicas y en este caso los EV, dentro de una didáctica que promueva una comprensión y construcción de los conceptos matemáticos.

Se ha planteado la importancia de estudiar cómo se incorporan las tecnologías en la enseñanza-aprendizaje de las matemáticas. Tanto los docentes como estudiantes juegan un papel muy importante en su incorporación, esto corresponde entonces a la capacitación del profesor de brindar las competencias necesarias en su enseñanza y el estudiante que tenga la capacidad de adquirirlas y ponerlas en práctica. En este sentido, Marques (2000) citado en Caballero (2009) discute la problemática de las competencias que los profesores deben desarrollar para que integren las tecnologías en sus clases, tanto virtuales como presenciales y afirma que la capacitación de los profesores en el uso de tecnologías digitales debe superar la simple capacitación en el conocimiento técnico de la herramienta tecnológica, debe implicar también el conocimiento de un modelo pedagógico asociado a la tecnología.

En este sentido, cuando se incorpora tecnologías en la enseñanza de la matemática los estudiantes experimentan un aprendizaje significativo, claro si se les da un uso 
apropiado a las tecnologías. Por ello, el uso de dispositivos tecnológicos y software educativo en la educación matemática apunta a una forma de innovación pedagógica basado en constructivismo sociointeracionista que, con recursos informáticos como los teléfonos celulares, tabletas y portátiles, se puede tomar la experiencia de educador y así entender las estrategias utilizadas por los estudiantes para resolver problemas matemáticos (Almeida, 2016). Además, el uso de teléfonos celulares, tabletas y portátiles en la enseñanza de las matemáticas son un medio de mejorar el rendimiento de los estudiantes, implicando un mundo virtual con herramientas capaces de estimular el interés en las matemáticas.

El uso de equipo tecnológico, que como ya se mencionó, se ha vuelto indispensable en la actualidad y se vuelve indispensable involucrarlo en la enseñanza de la matemática para lograr un aprendizaje significativo en los estudiantes. Los docentes tienen un reto enorme para hacer una enseñanza interesante y que cumpla con lo que demanda la sociedad.

\section{Metodología de investigación}

\subsection{Finalidad y objetivos del estudio}

Esta investigación tiene por objetivo general analizar las actitudes hacia el uso de las tecnologías en el aprendizaje de las matemáticas del estudiantado, tomando en consideración las variable género, edad y grado académico de los estudiantes, por tales razones, se proponen los objetivos específicos siguientes: (1) Identificar las actitudes hacia el uso de las tecnologías en el aprendizaje de las matemáticas del estudiantado; (2) Explicar la relación entre los factores actitudinales hacia el uso de las tecnologías en el aprendizaje de las matemáticas del estudiantado; (3) Comprobar si existen diferencias significativas entre género, edad y grado académico en relación con las actitudes hacia el uso de las tecnologías en el aprendizaje de las matemáticas del estudiantado.

\subsection{Metodología de la investigación}

Esta investigación se sustenta en el enfoque cuantitativo porque esta metodología a guiado el tratamiento de los datos a través de la categorización y descripción de las propiedades, características y perfiles de las personas, grupos, comunidades, procesos y objetos que se han sometidos a análisis (Hernández et al. 2014). El diseño de esta investigación es "Ex Post Facto" porque este tipo de investigación trata de descubrir fenómenos que ocurren en forma natural, pero miden diversas variables para analizar su posible efecto (Bisquerra, 2012). 


\subsection{Descripción de los participantes}

El estudio se llevó a cabo con una muestra de 158 estudiantes de diferentes instituciones educativas, pertenecientes a comunidades de los departamentos de Francisco Morazán, Intibucá, Valle y Yoro.

\begin{tabular}{|l|c|c|c|}
\hline \multicolumn{4}{|c|}{ Tabla 1. Participantes de la Investigación } \\
\hline \multicolumn{1}{|c|}{ Grado } & Mujeres & Hombres & Total \\
\hline Noveno & 27 & 19 & 46 \\
\hline Décimo & 46 & 24 & 70 \\
\hline Undécimo & 32 & 10 & 42 \\
\hline Total & 105 & 53 & 158 \\
\hline
\end{tabular}

El 66.5\% (105) de los participantes son mujeres, frente al 33.5\% (53) que son hombres. Respecto a su edad, la mayoría de los participantes se encuentran en el intervalo de $13-16$ años lo cual representa un $81.6 \%$ de la muestra. En referencia a la variable grado académico: el $29.1 \%$ son del grado noveno; $44.3 \%$ del grado décimo; $26.6 \%$ del undécimo grado. Otra característica de la muestra es que la mayoría de los estudiantes utilizan tecnologías de la información y comunicación para acceder a sus estudios virtuales. Para concluir, hay que indicar que el tipo de muestreo que hemos utilizado ha sido el muestreo no probabilístico causal o accidental, que es aquel en el cual el investigador selecciona directa e intencionalmente la muestra, debido fundamentalmente a que tiene fácil acceso a la misma y es representativa de la población (Gil, Rodríguez \& García, 1995; Albert, 2006; Sabariego, 2004).

\subsection{Instrumentos de recogida de datos}

El instrumento para la recopilación de la información fue el cuestionario de actitudes hacia el uso de las tecnologías en el aprendizaje de las matemáticas del estudiantado de López, Castro-Martínez y Molina (2013). Según Flores (2017) el instrumento está constituido por 35 ítems, aglutinados en 5 dimensiones. Los estudiantes pueden indicar su agrado de acuerdo o desacuerdo con las afirmaciones propuestas por media de una escala Likert de 5 valores. Los factores asociados son: interacción ordenador y matemáticas; actitudes hacia el aprendizaje de las matemáticas con tecnología; actitudes del uso de las tecnologías en el aprendizaje de las matemáticas; experiencias de matemáticas con tecnología; y matemática y tecnología.

\subsection{Procedimiento de administración y enfoque ético}

La administración del instrumento se realizó por parte de los autores durante el primer semestre 2021. Tenía un carácter anónimo y fue complementado por los sujetos participantes en presencia del profesorado. Con anterioridad a la toma de datos, 
se obtuvo tanto el consentimiento previo, libre e informado de los participantes, así como la autorización de las autoridades educativas.

\subsection{Análisis de datos}

Para satisfacer el objetivo del estudio se procedió al desarrollo de distintos análisis, empleando el paquete estadístico para las ciencias sociales SPSS v.25. Entre ellos, se aplicó un análisis de consistencia interna, un análisis descriptivo de los datos, un análisis de las correlaciones entre las categorías y pruebas paramétricas.

\section{Resultados}

La mayoría de los participantes (89.10\%; $M=3.65 ; \mathrm{SD}=1.07$ ) informaron estar muy de acuerdo / de acuerdo que las computadoras me ayudaran aprender mejora las matemáticas proporcionándome al instante muchos ejemplos de manera interactiva, además, las computadoras me ayudan a vincular el conocimiento, por ejemplo, la gráficas y ecuaciones (70.20\%; $M=4.01$; $S D=1.05$ ), sin embargo, a los estudiantes les resulta difícil comprender la transferencia de ideas de la pantalla de una computadora a su mente (55.10\%; $M=2.80 ; S D=1.29)$, así mismo, cuando leo en la pantalla de una computadora, tengo la tendencia a pasar por alto detalles de las ideas matemáticas (71.50\%; $\mathrm{M}=3.15 ; \mathrm{SD}=1.16)$, por ello, el no tener que preocuparme por los cálculos aritméticos, hace que me concentre mejor en las ideas esenciales de las matemáticas (81\%; $M=3.39 ; S D=1.09$ ). Entonces considero que el material impreso en la pantalla de una computadora y la copia impresa en el papel útiles para tomar notas (82.30\%; $M=4.26$; $S D=1.01$ ), por consiguiente, rara vez revisan el material inmediatamente después de que una sesión por computadora ha terminado (74.7\%; $M=3.36 ; S D=1.23$ ), entonces, el seguimiento de las instrucciones tecleadas pone atención fuera de las matemáticas (81\%; $M=3.12 ; S D=1.01)$. Es importante resaltar que la puntuación media en la interacción ordenador y matemáticas es $27.73(S D=4.31 ; S=18.65)$ para un $69 \%$ de la puntuación máxima de 40 puntos. Esto resultado significa que los estudiantes en el proceso de aprendizaje con las matemáticas interactúan con las computadoras.

\begin{tabular}{|c|c|c|c|c|c|c|}
\hline \multirow[b]{2}{*}{ Criterios } & \multicolumn{5}{|c|}{ Respuestas \% } & \multirow[b]{2}{*}{$\begin{array}{c}\text { Puntu- } \\
\text { ación } \\
\text { Media } \\
\text { (SD) }\end{array}$} \\
\hline & $\begin{array}{l}\text { Fuerte- } \\
\text { mente en } \\
\text { desacu- } \\
\text { erdo }\end{array}$ & $\begin{array}{c}\text { En desacu- } \\
\text { erdo }\end{array}$ & $\begin{array}{l}\text { In- } \\
\text { dife- } \\
\text { rente }\end{array}$ & $\begin{array}{c}\text { De acu- } \\
\text { erdo }\end{array}$ & $\begin{array}{l}\text { Fuerte- } \\
\text { mente de } \\
\text { acuerdo }\end{array}$ & \\
\hline $\begin{array}{l}\text { 2. Me resulta difícil comprender la transferencia } \\
\text { de ideas de la pantalla de una computadora a mi } \\
\text { mente. }\end{array}$ & 19.0 & 25.9 & 23.4 & 19.0 & 12.7 & $\begin{array}{c}2.80 \\
(1.299)\end{array}$ \\
\hline
\end{tabular}


Tabla 2. Interacción ordenador y matemáticas

\begin{tabular}{|c|c|c|c|c|c|c|}
\hline \multirow[b]{2}{*}{ Criterios } & \multicolumn{5}{|c|}{ Respuestas \% } & \multirow[b]{2}{*}{$\begin{array}{l}\text { Puntu- } \\
\text { ación } \\
\text { Media } \\
\text { (SD) }\end{array}$} \\
\hline & $\begin{array}{l}\text { Fuerte- } \\
\text { mente en } \\
\text { desacu- } \\
\text { erdo }\end{array}$ & $\begin{array}{l}\text { En desacu- } \\
\text { erdo }\end{array}$ & $\begin{array}{l}\text { In- } \\
\text { dife- } \\
\text { rente }\end{array}$ & $\begin{array}{l}\text { De acu- } \\
\text { erdo }\end{array}$ & $\begin{array}{l}\text { Fuerte- } \\
\text { mente de } \\
\text { acuerdo }\end{array}$ & \\
\hline $\begin{array}{l}\text { 3. El no tener que preocuparme por los cálculos } \\
\text { aritméticos, hace que me concentre mejor en las } \\
\text { ideas esenciales de las matemáticas. }\end{array}$ & 5.1 & 13.9 & 37.3 & 24.7 & 19.0 & $\begin{array}{c}3.39 \\
(1.098)\end{array}$ \\
\hline $\begin{array}{l}\text { 4. Cuando leo la pantalla de una computadora, } \\
\text { tengo la tendencia a pasar por alto detalles de las } \\
\text { ideas matemáticas }\end{array}$ & 9.5 & 19.0 & 32.9 & 24.7 & 13.9 & $\begin{array}{c}3.15 \\
(1.167)\end{array}$ \\
\hline $\begin{array}{l}\text { 5. Considero que el material impreso en la pan- } \\
\text { talla de una computadora y la copia impresa en el } \\
\text { papel es útil para tomar notas. }\end{array}$ & 4.4 & 0.6 & 12.7 & 29.1 & 53.2 & $\begin{array}{c}4.26 \\
(1.011)\end{array}$ \\
\hline $\begin{array}{l}\text { 6. Rara vez reviso el material inmediatamente } \\
\text { después de que una sesión por computadora ha } \\
\text { terminado. }\end{array}$ & 7.6 & 17.7 & 29.1 & 22.2 & 23.4 & $\begin{array}{c}3.36 \\
(1.232)\end{array}$ \\
\hline $\begin{array}{l}\text { 7. El seguimiento de las instrucciones tecleadas } \\
\text { pone atención fuera de las matemáticas. }\end{array}$ & 8.9 & 10.1 & 50.6 & 20.9 & 9.5 & $\begin{array}{c}3.12 \\
(1.018)\end{array}$ \\
\hline $\begin{array}{l}\text { 8. Las computadoras me ayudan a vincular el } \\
\text { conocimiento, como, por ejemplo, la forma de los } \\
\text { gráficos y sus ecuaciones. }\end{array}$ & 1.9 & 7.6 & 20.3 & 27.8 & 42.4 & $\begin{array}{c}4.01 \\
(1.053)\end{array}$ \\
\hline \multicolumn{7}{|l|}{ Puntuación Total a } \\
\hline Mediana (rango) & \multicolumn{6}{|l|}{$29(11-40)$} \\
\hline Media (SD) & \multicolumn{6}{|c|}{$27.73(4.31)$} \\
\hline Varianza & \multicolumn{6}{|l|}{$(18.65)$} \\
\hline Puntuación total \% & \multicolumn{6}{|l|}{$69 \%$} \\
\hline
\end{tabular}

a Máxima puntuación $=40$

La mayoría de los participantes (67.10\%; $M=3.27$; $S D=1.41)$ informaron estar muy de acuerdo / de acuerdo que les gusta usar computadora en matemáticas, además, afirman que vale la pena el esfuerzo adicional del uso de computadoras en matemáticas (84.7\%; $\mathrm{M}=3.75 ; \mathrm{SD}=1.23$ ), así mismo, las computadoras les ayudan a aprender mejor las matemáticas (71.15\%; $\mathrm{M}=3.21 ; \mathrm{SD}=1.29$ ), por consiguiente, las matemáticas son interesantes cuando se usa los ordenadores (84.20\%; $\mathrm{M=3.09;} S D=1.33$ ). Es importante mencionar que la puntuación media en el factor de actitudes hacia el aprendizaje de las matemáticas con tecnología es $13.32(S D=4.48 ; S=20.14)$ para un 66\% de la puntuación máxima de 20 puntos. Esto resultado representa que los estudiantes tienen actitudes positivas hacia el aprendizaje de las matemáticas con tecnologías. 


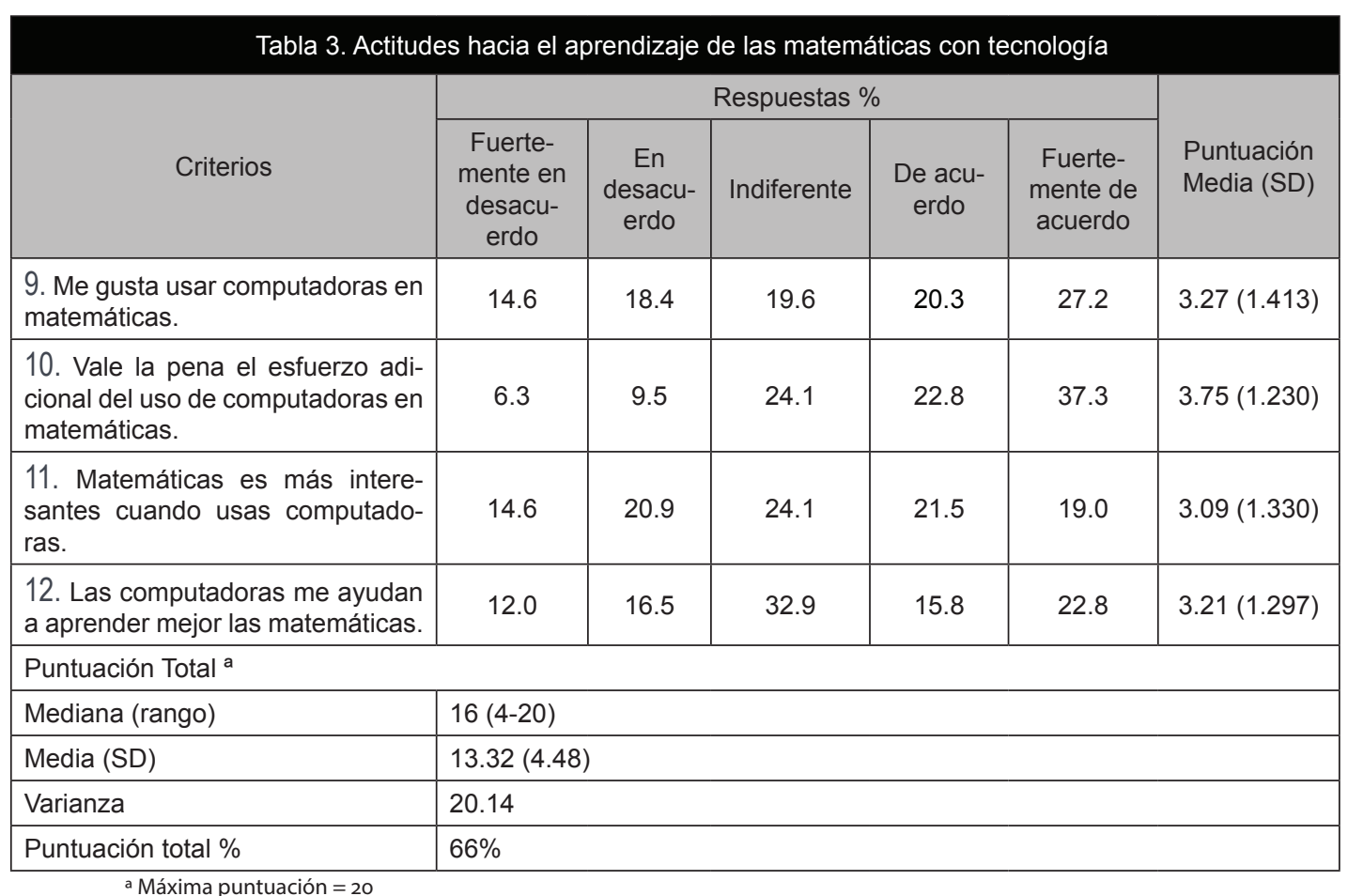

a Máxima puntuación $=20$

La mayoría de los participantes (54.40\%; $M=3.63 ; \mathrm{SD}=1.164)$ informaron estar muy de acuerdo / de acuerdo que el poder la computación haca más fácil explorar ideas matemáticas, además, las computadoras están cambiando el modo de hacer matemáticas (55.10\%; $M=3.44 ; S D=1.23)$; el uso de la tecnología para cálculos me facilita hacer las aplicaciones más realísticas (51.20\%; $\mathrm{M}=3.59$; $\mathrm{SD}=1.09$ ); por ello, nos gusta explorar métodos matemáticos e ideas usando tecnología (61.40\%; $M=3.70 ; S D=1.13$ ); y disponer de la tecnología para hacer el trabajo rutinario me permite probar diferentes métodos y enfoques (57.02\%; $M=3.62$; $S D=1.06$ ); aunque quiero mejorar en el uso de las computadoras para que me ayude en las matemáticas (70.90\%; $\mathrm{M}=4.05 ; \mathrm{SD}=1.03$ ) ya que, los símbolos y el lenguaje de las matemáticas son bastantes complicados sin la adición de la tecnología (75.40\%; $M=3.21 ; S D=1.17)$. Sin embargo, los estudiantes afirman que las computadoras son importantes, pero no sienten la necesidad de usarlas para aprender matemáticas (77.20\%; $\mathrm{M}=3.42$; $\mathrm{SD}=1.30$ ); las computadoras son buenas herramientas para los cálculos, pero no para mi aprendizaje de matemáticas (72.20\%; $\mathrm{M}=3.25 ; \mathrm{SD}=1.23$ ); igualmente, piensan que el uso de la tecnología es una pérdida de tiempo en el aprendizaje de las matemáticas (51.9\%; $\mathrm{M}=2.63 ; \mathrm{SD}=1.32)$, por consiguiente, prefieren hacer todos los cálculos y gráficos manualmente sin tener que usar una computadora (74.10\%; $\mathrm{M}=3.46 ; \mathrm{SD}=1.36$ ). Es importante mencionar que la puntuación media en el factor de actitudes del uso de la tecnología en el aprendizaje de las matemáticas es 37.98 ( $\mathrm{SD}=6.24 ; \mathrm{S}=39.04$ ) para un $69 \%$ de la puntuación máxima de 55 puntos. Esto resultado representa que los estudiantes tienen actitudes positivas hacia el aprendizaje de las matemáticas. 
Tabla 4. Actitudes del uso de la tecnología en el aprendizaje de las matemáticas

\begin{tabular}{|c|c|c|c|c|c|c|}
\hline \multirow[b]{2}{*}{ Criterios } & \multicolumn{5}{|c|}{ Respuestas \% } & \multirow[b]{2}{*}{$\begin{array}{l}\text { Puntuación } \\
\text { Media (SD) }\end{array}$} \\
\hline & $\begin{array}{l}\text { Fuerte- } \\
\text { mente en } \\
\text { desacu- } \\
\text { erdo }\end{array}$ & $\begin{array}{l}\text { En } \\
\text { desacu- } \\
\text { erdo }\end{array}$ & $\begin{array}{l}\text { Indife- } \\
\text { rente }\end{array}$ & $\begin{array}{l}\text { De acu- } \\
\text { erdo }\end{array}$ & $\begin{array}{l}\text { Fuerte- } \\
\text { mente de } \\
\text { acuerdo }\end{array}$ & \\
\hline $\begin{array}{l}\text { 13. El poder de la computación hace más } \\
\text { fácil explorar ideas matemáticas. }\end{array}$ & 5.1 & 10.8 & 29.7 & 24.7 & 29.7 & $3.63(1.164)$ \\
\hline $\begin{array}{l}\text { 14. Las computadoras están cambiando } \\
\text { el modo de hacer matemáticas. }\end{array}$ & 9.5 & 12.7 & 22.8 & 34.2 & 20.9 & $3.44(1.223)$ \\
\hline $\begin{array}{l}\text { 15. Sé que las computadoras son impor- } \\
\text { tantes, pero no siento la necesidad de us- } \\
\text { arlas para aprender matemáticas. }\end{array}$ & 11.4 & 11.4 & 27.8 & 22.8 & 26.6 & $3.42(1.303)$ \\
\hline $\begin{array}{l}\text { 16. Las computadoras son buenas herra- } \\
\text { mientas para los cálculos, pero no para mi } \\
\text { aprendizaje de matemáticas. }\end{array}$ & 8.9 & 19.0 & 31.0 & 20.3 & 20.9 & $3.25(1.236)$ \\
\hline $\begin{array}{l}\text { 17. Pienso que el uso de la tecnología es } \\
\text { una pérdida de tiempo en el aprendizaje } \\
\text { de las matemáticas. }\end{array}$ & 26.6 & 21.5 & 25.9 & 14.6 & 11.4 & $2.63(1.323)$ \\
\hline $\begin{array}{l}\text { 18. Prefiero hacer todos los cálculos y } \\
\text { gráficos manualmente sin tener que usar } \\
\text { una computadora. }\end{array}$ & 10.8 & 15.2 & 24.1 & 17.7 & 32.3 & $3.46(1.362)$ \\
\hline $\begin{array}{l}\text { 19. El uso de la tecnología para cálculos } \\
\text { me facilita hacer las aplicaciones más re- } \\
\text { alísticas. }\end{array}$ & 5.1 & 7.0 & 36.7 & 25.9 & 25.3 & $3.59(1.095)$ \\
\hline $\begin{array}{l}\text { 20. Me gustan explorar métodos } \\
\text { matemáticos e ideas usando tecnología. }\end{array}$ & 3.8 & 12.7 & 22.2 & 32.3 & 29.1 & $3.70(1.132)$ \\
\hline $\begin{array}{l}\text { 21. Disponer de la tecnología para hacer } \\
\text { el trabajo rutinario me permite probar dife- } \\
\text { rentes métodos y enfoques. }\end{array}$ & 4.4 & 8.9 & 29.7 & 34.2 & 22.8 & $3.62(1.068)$ \\
\hline $\begin{array}{l}\text { 22. Quiero mejorar en el uso de las com- } \\
\text { putadoras para que me ayude en las } \\
\text { matemáticas. }\end{array}$ & 1.9 & 6.3 & 20.9 & 26.6 & 44.3 & $4.05(1.039)$ \\
\hline $\begin{array}{l}\text { 23. Los símbolos y el lenguaje de las } \\
\text { matemáticas son bastantes complicados } \\
\text { sin la adición de la tecnología. }\end{array}$ & 10.8 & 13.3 & 34.2 & 26.6 & 14.6 & $3.21(1.177)$ \\
\hline \multicolumn{7}{|l|}{ Puntuación Total ${ }^{a}$} \\
\hline Mediana (rango) & \multicolumn{6}{|l|}{$39(16-55)$} \\
\hline Media (SD) & \multicolumn{6}{|c|}{$37.98(6.24)$} \\
\hline Varianza & \multicolumn{6}{|l|}{39.04} \\
\hline Puntuación total \% & \multicolumn{6}{|l|}{$69 \%$} \\
\hline
\end{tabular}

a Máxima puntuación = 55

La mayoría de los participantes (69.6o\%; $M=3.13$; $S D=1.26)$ informaron estar muy de acuerdo / de acuerdo que los softwares son útiles para mi aprendizaje de las matemáticas; por ello, el uso de software hace el aprendizaje de las matemáticas más interesantes (78.50\%; $\mathrm{M}=3.36 ; \mathrm{SD}=1.12)$; en términos generales vale la pena aprender a 
utilizar el software para hacer matemáticas (61.30\%; $M=3.80 ; S D=1.13$ ); por consiguiente, por propia elección usaré las veces que sea necesario software para matemáticas (73.90; $\mathrm{M}=3.31 ; \mathrm{SD}=1.28$ ); aunque aprender a usar software para hacer matemáticas es frustrante (67.10; $M=2.96 ; S D=1.13$ ). Es importante mencionar que la puntuación media en el factor de experiencias matemáticas con tecnologías es $16.56(\mathrm{SD}=4.10$; $S=16.84$ ) para un $66 \%$ de la puntuación máxima de 25 puntos. Esto resultado representa que los estudiantes tienen buenas experiencias matemáticas con tecnologías.

\begin{tabular}{|c|c|c|c|c|c|c|}
\hline \multirow[b]{2}{*}{ Criterios } & \multicolumn{5}{|c|}{ Respuestas \% } & \multirow[b]{2}{*}{$\begin{array}{l}\text { Puntuación } \\
\text { Media (SD) }\end{array}$} \\
\hline & $\begin{array}{l}\text { Fuerte- } \\
\text { mente en } \\
\text { desacu- } \\
\text { erdo }\end{array}$ & $\begin{array}{l}\text { En de- } \\
\text { sacuerdo }\end{array}$ & $\begin{array}{l}\text { Indife- } \\
\text { rente }\end{array}$ & $\begin{array}{l}\text { De acu- } \\
\text { erdo }\end{array}$ & $\begin{array}{l}\text { Fuerte- } \\
\text { mente de } \\
\text { acuerdo }\end{array}$ & \\
\hline $\begin{array}{l}\text { 24. He encontrado software útil para mi } \\
\text { aprendizaje de las matemáticas }\end{array}$ & 13.3 & 17.1 & 29.7 & 22.8 & 17.1 & $3.13(1.267)$ \\
\hline $\begin{array}{l}\text { 25. Aprender a usar software para hac- } \\
\text { er matemáticas es frustrante. }\end{array}$ & 10.8 & 22.2 & 38.6 & 17.1 & 11.4 & $2.96(1.134)$ \\
\hline $\begin{array}{l}\text { 26. El uso de software hace el apre- } \\
\text { ndizaje de las matemáticas más inte- } \\
\text { resantes. }\end{array}$ & 5.7 & 15.8 & 33.5 & 26.6 & 18.4 & $3.36(1.124)$ \\
\hline $\begin{array}{l}\text { 27. En términos generales vale la pena } \\
\text { aprender a utilizar el software para } \\
\text { hacer matemáticas. }\end{array}$ & 3.8 & 9.5 & 25.3 & 25.9 & 35.4 & $3.80(1.139)$ \\
\hline $\begin{array}{l}\text { 28. Por propia elección usaré las vec- } \\
\text { es que sea necesario software para } \\
\text { matemáticas. }\end{array}$ & 11.4 & 14.6 & 27.8 & 24.1 & 22.2 & $3.31(1.281)$ \\
\hline \multicolumn{7}{|l|}{ Puntuación Total a } \\
\hline Mediana (rango) & \multicolumn{6}{|l|}{$20(5-25)$} \\
\hline Media (SD) & \multicolumn{6}{|l|}{$16.56(4.10)$} \\
\hline Varianza & \multicolumn{6}{|l|}{16.84} \\
\hline Puntuación total \% & \multicolumn{6}{|l|}{$66 \%$} \\
\hline
\end{tabular}

a Máxima puntuación $=25$

La mayoría de los participantes (51.30\%; $\mathrm{M}=3.41 ; \mathrm{SD}=1.23$ ) informaron estar muy de acuerdo / de acuerdo que les gusta aprender matemáticas con la ayuda de la computadora; porque las tareas matemáticas por computadora son claras y fáciles de leer (62\%; $\mathrm{M}=3.77 ; \mathrm{SD}=1.13$ ); además, la retroalimentación inmediata de la computadora es útil para resolver problemas matemáticos (60.10\%; $M=3.70 ; S D=1.09)$; ya que la revisión de la lección en la tarea por computadora me ayuda a repasar los conceptos matemáticos (57.60\%; $\mathrm{M}=3.75 ; \mathrm{SD}=1.08$ ); y los exámenes de matemáticas por computadoras con puntuación inmediata me ayudan a evaluar mi propio entendimiento y rendimiento (73.40\%; $\mathrm{M}=4.06 ; \mathrm{SD}=1.02)$; en definitiva, les gustan las pruebas de matemáticas por computadoras más que las pruebas de lápiz y papel $(\mathrm{M}=63.40 \% ; 18$; $\mathrm{SD}=1.52)$. Es importante mencionar que la puntuación media en el factor de matemáticas y tecnologías es $21.87(S D=5.21 ; S=27.23)$ para un $72 \%$ de la puntuación máxima 
de 30 puntos. Esto resultado representa que los estudiantes tienen buena motivación entre el uso de las matemáticas con tecnologías.

\begin{tabular}{|c|c|c|c|c|c|c|}
\hline \multirow[b]{2}{*}{ Criterios } & \multicolumn{5}{|c|}{ Respuestas \% } & \multirow[b]{2}{*}{$\begin{array}{l}\text { Puntuación } \\
\text { Media (SD) }\end{array}$} \\
\hline & $\begin{array}{l}\text { Fuerte- } \\
\text { mente en } \\
\text { desacu- } \\
\text { erdo }\end{array}$ & $\begin{array}{c}\text { En de- } \\
\text { sacuerdo }\end{array}$ & $\begin{array}{l}\text { Indife- } \\
\text { rente }\end{array}$ & $\begin{array}{l}\text { De acu- } \\
\text { erdo }\end{array}$ & $\begin{array}{l}\text { Fuerte- } \\
\text { mente de } \\
\text { acuerdo }\end{array}$ & \\
\hline $\begin{array}{l}\text { 29. Me gusta aprender matemáticas con la } \\
\text { ayuda de la computadora. }\end{array}$ & 8.2 & 16.5 & 24.1 & 28.5 & 22.8 & $3.41(1.237)$ \\
\hline $\begin{array}{l}\text { 30. Las tareas matemáticas por computa- } \\
\text { dora son claras y fáciles de leer. }\end{array}$ & 5.1 & 7.6 & 25.3 & 29.7 & 32.3 & $3.77(1.135)$ \\
\hline $\begin{array}{l}\text { 31. La retroalimentación inmediata de la } \\
\text { computadora es útil para resolver problemas } \\
\text { matemáticos. }\end{array}$ & 3.8 & 10.1 & 25.9 & 32.9 & 27.2 & $3.70(1.095)$ \\
\hline $\begin{array}{l}\text { 32. La revisión de la lección en la tarea por } \\
\text { computadora me ayuda a repasar los con- } \\
\text { ceptos matemáticos }\end{array}$ & 1.9 & 10.8 & 29.7 & 25.3 & 32.3 & $3.75(1.081)$ \\
\hline $\begin{array}{l}\text { 33. Los exámenes de matemáticas por com- } \\
\text { putadoras con puntuación inmediata me } \\
\text { ayudan a evaluar mi propio entendimiento y } \\
\text { rendimiento. }\end{array}$ & 2.5 & 5.1 & 19.0 & 30.4 & 43.0 & $4.06(1.026)$ \\
\hline $\begin{array}{l}\text { 34. Me gustan las pruebas de matemáticas } \\
\text { por computadoras más que las pruebas de } \\
\text { lápiz y papel. }\end{array}$ & 20.3 & 16.5 & 18.4 & 14.6 & 30.4 & $3.18(1.522)$ \\
\hline \multicolumn{7}{|l|}{ Puntuación Total $^{a}$} \\
\hline Mediana (rango) & \multicolumn{6}{|l|}{$23(7-30)$} \\
\hline Media (SD) & \multicolumn{6}{|c|}{$21.87(5.21)$} \\
\hline Varianza & \multicolumn{6}{|l|}{27.23} \\
\hline Puntuación total \% & \multicolumn{6}{|l|}{$72 \%$} \\
\hline
\end{tabular}

Se puede verificar en la tabla 7, que existe una correlación significativa entre cada una de las categorías analizadas en este estudio mostrando una relación directamente proporcional entre ellas. Por ejemplo, las actitudes hacia el aprendizaje de las matemáticas con tecnología; y matemáticas y tecnologías $(r=0.681)$; esto significa que a medida que exista una enseñanza de las matemáticas con tecnología las actitudes positivas de los estudiantes hacia el aprendizaje de éstas incrementan. También, las experiencias matemáticas con tecnologías se relacionan con las matemáticas y tecnologías $(r=0.599)$, igualmente, se observa que la interacción ordenador y matemáticas ayuda incrementar las actitudes del uso de tecnologías en el aprendizaje de las matemáticas $(r=0.563)$, esto se debe a las potenciales prácticas matemáticas con tecnologías $(r=0.552)$. 
Tabla 7: Análisis de correlaciones entre las categorías ( $r$-Pearson)

\begin{tabular}{|l|c|c|c|c|c|}
\hline \multicolumn{1}{|c|}{ Factores } & Categoría 1 & Categoría 2 & Categoría 3 & Categoría 4 & Categoría 5 \\
\hline $\begin{array}{l}\text { 1. Interacción ordenador y } \\
\text { matemáticas. }\end{array}$ & 1 & 0.475 & 0.563 & 0.477 & 0.378 \\
\hline $\begin{array}{l}\text { 2. Actitudes hacia el apren- } \\
\text { dizaje de las matemáticas con } \\
\text { tecnología. }\end{array}$ & & 1 & 0.311 & 0.467 & 0.681 \\
\hline $\begin{array}{l}\text { 3. Actitudes del uso de la tec- } \\
\text { nología en el aprendizaje de } \\
\text { las matemáticas }\end{array}$ & & 1 & 0.552 & 0.384 \\
\hline $\begin{array}{l}\text { 4. Experiencias matemáticas } \\
\text { con tecnologías }\end{array}$ & & & & 1 & 0.599 \\
\hline 5. Matemáticas y tecnologías. & & & & & 1 \\
\hline
\end{tabular}

Se realizó un análisis no paramétrico en función de las variables: género, edad, grado académico, tipo de escuela, modalidad de estudio, y tipo de tecnología con la finalidad de establecer si existen diferencias significativas entre estas variables y los factores actitudinales hacia las matemáticas con tecnologías. En este sentido, se puede apreciar los resultados de las pruebas paramétricas (2 muestras independientes - U de Mann - Whitney; K muestras independientes H de Kruskal - Wallis):

\begin{tabular}{|l|c|c|c|c|c|c|}
\hline \multicolumn{1}{|c|}{ Factores } & Género & Edad & $\begin{array}{c}\text { Grado Aca- } \\
\text { démico }\end{array}$ & $\begin{array}{c}\text { Tipo de } \\
\text { Escuela }\end{array}$ & $\begin{array}{c}\text { Modalidad de } \\
\text { Estudio }\end{array}$ & $\begin{array}{c}\text { Tipo de } \\
\text { tecnología }\end{array}$ \\
\cline { 2 - 7 } & $p$ & $p$ & $p$ & $p$ & $p$ & $P$ \\
\hline $\begin{array}{l}\text { Interacción ordenador y } \\
\text { matemáticas. }\end{array}$ & 0.647 & 0.704 & 0.900 & 0.001 & 0.916 & 0.023 \\
\hline $\begin{array}{l}\text { Actitudes hacia el apren- } \\
\text { dizaje de las matemáticas } \\
\text { con tecnología. }\end{array}$ & 0.118 & 0.346 & 0.482 & 0.000 & 0.274 & 0.520 \\
\hline $\begin{array}{l}\text { Actitudes del uso de la tec- } \\
\text { nología en el aprendizaje } \\
\text { de las matemáticas }\end{array}$ & 0.782 & 0.516 & 0.470 & 0.009 & 0.787 & 0.004 \\
\hline $\begin{array}{l}\text { Experiencias matemáticas } \\
\text { con tecnologías }\end{array}$ & 0.225 & 0.736 & 0.620 & 0.001 & 0.568 & 0.513 \\
\hline Matemáticas y tecnologías. & 0.207 & 0.401 & 0.220 & 0.002 & 0.149 & 0.799 \\
\hline
\end{tabular}

Los resultados de ambas pruebas significan lo siguiente:

- El género no influye en los factores actitudes hacia el uso de las tecnologías en el aprendizaje de las matemáticas del estudiantado porque el valor de la significancia es mayor que 0.05. Esto significa que hombres y mujeres tienen las mismas actitudes hacia el uso de las tecnologías en el aprendizaje de las matemáticas. 
- La edad no es determinante en los factores actitudes hacia el uso de las tecnologías en el aprendizaje de las matemáticas del estudiantado porque el valor de la significancia es mayor que 0.05 . Esto significan que todos los participantes de las distintas edades tienen las mismas percepciones hacia el uso de tecnologías en las matemáticas.

- El grado académico no es concluyente en los factores actitudes hacia el uso de las tecnologías en el aprendizaje de las matemáticas del estudiantado porque el valor de la significancia es mayor que 0.05. Todos los participantes procedentes de los diferentes grados académicos tienen las mismas actitudes hacia el uso de las tecnologías en las matemáticas.

- La modalidad de estudio no es categórica en los factores actitudes hacia el uso de las tecnologías en el aprendizaje de las matemáticas del estudiantado porque el valor de la significancia es mayor que 0.05 . Todos los participantes procedentes de las diferentes modalidades tienen las mismas actitudes hacia el uso de las tecnologías en las matemáticas.

- El tipo de tecnología no es decisiva en los factores: (1) actitudes hacia el aprendizaje de las matemáticas con tecnología; (2) experiencias matemáticas con tecnologías; y (3) matemáticas y tecnologías porque el valor de la significancia es mayor que 0.05 , esto significa que los participantes usan en las mismas condiciones las computadoras y teléfonos inteligentes. Sin embargo, existen diferencias en los factores de interacción ordenador y matemáticas; y actitudes del uso de la tecnología en el aprendizaje de las matemáticas porque el valor de la significancia es menor que 0.05. Esto quiere decir, que el uso del teléfono inteligente es determinante en el aprendizaje de las matemáticas.

\section{Discusión y conclusiones}

En esta investigación se ha analizado las actitudes hacia el uso de las tecnologías en el aprendizaje de las matemáticas del estudiantado. Es importante mencionar que la identificación de dichas actitudes contribuye en gran manera en la mejora de la enseñanza y el aprendizaje de las matemáticas, ya que de esta forma el docente verifica si el proceso se vuelve más motivador y entretenido para sus estudiantes, tanto que hasta busquen por mi mismos seguir aprendiendo y actualizándose.

Se encontró que existe interacción entre ordenador y matemáticas $(M=27.73$; $\mathrm{SD}=4.31 ; \mathrm{S}=18.65$ ) por parte de este grupo de estudiantes, coincidiendo con los estudios de Flores-López (2017) que afirman que estas interacciones son entendidas con como la vinculación del conocimiento, así mismo, concuerdan con los resultados del Galbraith y Haines (2000) que declararon que los estudiantes mejoran su aprendizaje 
cuando interactúan con la computadora. Por otra parte, se descubrieron actitudes positivas hacia el aprendizaje de las matemáticas con tecnología $(M=13.32 ; S D=4.48$; $S=20.14$ ), estas actitudes son comprendidas como el esfuerzo adicional del uso de las computadoras en matemáticas (Flores-López, 2017), igualmente, las tecnologías ofrecen nuevos paradigmas para la enseñanza (Pierce et al., 2007) por consiguiente, es necesario una enseñanza disruptiva en el aprendizaje de las matemáticas (FloresLópez, 2020).

Las actitudes hacia el uso de la tecnología en el aprendizaje de las matemáticas $(\mathrm{M}=37.98 ; \mathrm{SD}=6.24 ; \mathrm{S}=39.04)$ son positivas, estos resultados se relacionan con los estudios de Flores-López (2017) que afirman que disponer de tecnologías en la enseñanza de las matemáticas facilita la construcción colectiva de las soluciones de un problema. Además, las experiencias matemáticas con tecnologías $(M=16.56 ; S D=4.10$; $S=16.84$ ) son notables con buenas prácticas matemáticas vinculadas a la resolución de problemas (Flores-López, 2021). Finalmente, las matemáticas y tecnologías ( $M=21.87$; $S D=5.21 ; S=27.23)$ se muestra una buena motivación entre uso de las matemáticas con tecnologías. Esto se vincula con los resultados de Flores-López y Auzmendi (2018) que indican que los estudiantes se sienten motivados en el aprendizaje de las matemáticas.

En relación con las variables género, edad, grado académico, tipo de escuela, modalidad de estudio y tipo de tecnología se encontró que no existen diferencias significativas coincidiendo con los estudios de Flores-López (2017) y Flores-López y Auzmendi (2018) que no encontraron diferencias significativas entre dichas variables, igualmente, afirman que estas actitudes se manifiestan de manera relevante entre el tipo de dispositivo tecnológico (computador y teléfono inteligente), lo que indican que los estudiantes en su mayoría interaccionan más con los teléfonos inteligente al momento de resolver prácticas matemáticas. Se puede concluir, que el aprendizaje de las matemáticas con tecnologías constituye un elemento disruptivo para la enseñanza de las matemáticas porque el estudiante es quien va a seguir interactuando con dicha disciplina no solo en el ámbito académico, sino también en el profesional (Flores-López \& Auzmendi, 2018).

\section{Lista de referencia}

Albert-Gómez, M. J. (2006). La investigación educativa: Claves Teóricas. McGrawHill.https://www.academia.edu/download/59095661/LA_INVESTIGACION_ EDUCATIVA._CLAVES_TEORICAS20190430-128070-a4elay.PDF

Almedia, H. (2016). El uso de teléfonos celulares, tabletas y portátiles en la enseñanza de las matemáticas. Revista científica multidisciplinaria base de conocimiento, 9, 829-814. Recuperada de: https://www.nucleodoconhecimento.com.br/ educacion-es/celulares-tablets-ensenar-matematicas 
Bisquerra-Alzina, R. (2012). Metodología de la investigación educativa. La Muralla.

Caballero, A. (2009). Cambios en la enseñanza de las matemáticas al incorporar tecnologías digitales al taller de computación de una escuela telesecundaria. (Tesis de pregrado). Centro De Investigación Y De Estudios Avanzados Del Instituto Politécnico Nacional, México. Recuperado de: http://www.matedu.cinvestav. $\mathrm{mx} /$ asacristan/Tesistas/AcrisioCaballeroTesis.pdf

Flores López, W. O. (2017). Incorporación de tecnologías en la enseñanza de las matemáticas. Horizontes Pedagógicos, 19(1), 21-30. https://doi.org/10.33881/01238264.hop.19103

Flores-López, W. O. (2020). La educación y la investigación ante el COVID-19: Aprendizaje para todos a lo largo de toda la vida. Revista Electrónica de Conocimientos, Saberes y Prácticas, 3(2). https://doi.org/10.5377/recsp.v3i2.10685

Flores-López, W.O \& Auzmendi, E. (2018). Actitudes hacia las matemáticas en la enseñanza universitaria y su relación con las variables género y etnia. Profesorado. Revista de Currículum y Formación de Profesorado, 22(3), 231-251. https://doi. org/10.30827/profesorado.v22i3.8000

Galbraith, P., \& Haines, C. (200o). Mathematics-computing Attitudes Scales. Monographs in Continuing Education. London: University London.

Gil, J., Rodríguez, G. y García, E. (1995). Estadística básica aplicada a las ciencias de la educación. Kronos.

Hernández-Sampieri, R., Fernández-Collado, C. y Baptista-Lucio, M. (2014). Metodología de la investigación. McGraw-Hill.

López, R., Castro, E., \& Molina, M. (2013). Actitudes de estudiantes de ingeniería de nuevo ingreso hacia el uso de la tecnologia en matemáticas. PNA, 31-50

Pierce, R., Stacey, K., \& Barkatsas, A. (2007). A scale for monitoring students' attitudes to learning mathematics with technology. Computers \& Education, 16(2), 285-300.

Rojano, T. (2003). Incorporación de entornos tecnológicos de aprendizaje a la cultura escolar: proyecto de innovación educativa en matemáticas y ciencias en escuelas secundarias públicas de México. Revista Iberoamericana de Educación, 33, Recuperada de: https://rieoei.org/historico/documentos/rie3zao7.htm 
Sabariego, M. (2004). El proceso de investigación. En R. Bisquerra-Alzina, Metodología de la investigación educativa (127-163). La Muralla.

Salat-Figols, R. S. (2013). La enseñanza de las matemáticas y la tecnología. Innovación educativa (México, DF), 13(62), 61-74. 